\title{
A PCR-based strategy to generate integrative targeting alleles with large regions of homology
}

\author{
Robert C. Davidson, Jill R. Blankenship, Peter R. Kraus, Marisol de \\ Jesus Berrios, Christina M. Hull, Cletus D'Souza, Ping Wang \\ and Joseph Heitman
}

Author for correspondence: Joseph Heitman. Tel: +1 919684 2824. Fax: +1 9196845458. e-mail: heitm001@duke.edu

Departments of Molecular Genetics and Microbiology, Pharmacology and Cancer Biology, and Medicine, and the Howard Hughes Medical Institute, Duke University Medical Center, Research Drive, Durham, NC 27710, USA

\begin{abstract}
Cryptococcus neoformans is an opportunistic fungal pathogen with a defined sexual cycle for which genetic and molecular techniques are well developed. The entire genome sequence of one $C$. neoformans strain is nearing completion. The efficient use of this sequence is dependent upon the development of methods to perform more rapid genetic analysis including gene-disruption techniques. A modified PCR overlap technique to generate targeting constructs for gene disruption that contain large regions of gene homology is described. This technique was used to disrupt or delete more than a dozen genes with efficiencies comparable to those previously reported using cloning technology to generate targeting constructs. Moreover, it is shown that disruptions can be made using this technique in a variety of strain backgrounds, including the pathogenic serotype A isolate $\mathrm{H99}$ and recently characterized stable diploid strains. In combination with the availability of the complete genomic sequence, this gene-disruption technique should pave the way for higher throughput genetic analysis of this important pathogenic fungus.
\end{abstract}

Keywords: gene disruption, PCR overlap, Cryptococcus neoformans

\section{INTRODUCTION}

Cryptococcus neoformans is a basidiomycetous fungus that has been recognized as a human pathogen for more than a century (Casadevall \& Perfect, 1998; Sanfelice, 1894). C. neoformans mainly infects immunocompromised individuals, and disease typically manifests as meningoencephalitis (Casadevall \& Perfect, 1998). With the relative increase in recent years in the numbers of immunocompromised patients due to AIDS, cancer chemotherapy, transplant-rejection suppression therapy, and other factors, the incidence of opportunistic fungal infections has risen (Mitchell \& Perfect, 1995). C. neoformans infection results from inhalation of spores or desiccated yeast cells, which then spread via the bloodstream to infect the central nervous system and cause life-threatening meningoencephalitis.

In addition to being an important cause of disease in

Abbreviation: MAP, mitogen-acitvated protein. immunocompromised patients, C. neoformans is an excellent model of fungal pathogenesis. Several welldefined virulence factors include the polysaccharide capsule (which promotes intracellular survival in macrophages), the pigment melanin (which prevents oxidation by macrophages), the production of the enzymes urease and phospholipase $\mathrm{B}$, and the ability to grow at $37^{\circ} \mathrm{C}$ (Alspaugh et al., 2000a; Casadevall \& Perfect, 1998; Chen et al., 2000; Cox et al., 2000, 2001; Cruz et al., 2000; Fox et al., 2001; Kwon-Chung \& Bennett, 1992; Odom et al., 1997). Several animal models have been developed, including a mouse tail-vein injection model, rat and mouse inhalation models, and a rabbit meningitis model (Goldman et al., 1994; Kwon-Chung et al., 1982; Perfect et al., 1980). Importantly, C. neoformans is genetically tractable, with a well-characterized sexual cycle involving mating between two haploid mating types, MAT a and MAT $\alpha$ (Alspaugh et al., 2000b; KwonChung, 1975, 1976). Efficient transformation and genedisruption protocols have been developed along with auxotrophic and dominant selectable markers and 
overexpression plasmids (Davidson et al., 2000; Edman \& Kwon-Chung, 1990; Hua et al., 2000; McDade \& Cox, 2001; Sudarshan et al., 1999; Toffaletti et al., 1993). Furthermore, genetic analysis has been facilitated by the recent characterization of a stable diploid state, which allows identification and analysis of essential genes (Sia et al., 2000). Finally, the entire genome sequence is nearing completion for the serotype $\mathrm{D}$ strain JEC21, and a sequencing project has been initiated for the clinical serotype A isolate H99 (Stanford, TIGR, UBC, Duke, Whitehead; reviewed by Heitman et al., 1999). These advances will allow extensive comparative genomics experimentation between $C$. neoformans strains and other fungal genomes.

The efficient use of the complete genome sequence will require more rapid gene-function testing, a process that is limited by tedious construction of targeting alleles by cloning. PCR-generated targeting alleles have been employed for Saccharomyces cerevisiae and for the human pathogen Candida albicans, which has overcome the necessity for cloning and has allowed more highthroughput genetic analysis (Baudin et al., 1993; Eberhardt \& Hohmann, 1995; Lorenz et al., 1995; Wach, 1996; Wach et al., 1994; Wilson et al., 1999). However, efficient homologous recombination in C. neoformans requires larger regions of homology, which has prevented the application of similar PCR-based strategies.

Here we present a modified application of a technique called PCR overlap to generate targeting alleles with larger regions of homology (Ho et al., 1989; Horton et al., 1989). This technique can be used in any system where homologous recombination requires longer regions than those that can be incorporated into synthetic oligonucleotides, and will effectively eliminate the timeconsuming process of searching for convenient restriction sites and cloning targeting alleles.

\section{METHODS}

Gene disruptions. To create the ste $11 \alpha$ mutant strain, first the $\sim 4.5 \mathrm{~kb} S T E 11 \alpha$ locus was amplified from MAT $\alpha$ genomic DNA using primers JOHE5391 (GCTCGTTCTCCCCTGTAC) and JOHE5392 (CTGCCGACGCCGTGTAAT) (R. C. Davidson and others, unpublished). The ste $11 \alpha \Delta:$ :URA5 disruption allele was constructed using PCR overlap (outlined in Fig. 1). In the first round, the $5^{\prime}$ end of the $S T E 11 \alpha$ gene was amplified with primers JOHE5391 (primer 1) (GCTCGTTCTCCCCTGTAC) and JOHE5306 (primer 3) (GGTCGAGCAACTTCCTCATTTACAGGGCTGTCCTG), the $3^{\prime}$ end of the STE11 $\alpha$ gene with primers JOHE5307 (primer 4) (CCACCTCCTGGAGGCAAGACAGGGATATCAAAGGCG) and JOHE5392 (primer 6) (CTGCCGACGCCGTGTAAT), and the URA5 gene with primers JOHE5305 (primer 2) (CAGGACAGCCCTGTAAATGAGCGAAGTTGCTCGAAC) and JOHE5308 (primer 5) (CGCCTTTGATATCCCTGTCTTGCCTCCAGGAGGTGG). The bold text represents the fragment of the oligo corresponding to the selectable marker. The amplified products were run on a $0.6 \%$ agarose gel and extracted together using the Qiaquik column method (Qiagen). Primers JOHE5391 (no. 1) and JOHE5392 (no. 6) were then used to overlap the three products to yield the $4.2 \mathrm{~kb}$ ste $11 \alpha \Delta$ : URA5 disruption allele. The PCR product was gelpurified, extracted and directly introduced into the serotype D ura5 strain JEC43 by biolistic transformation.

The tor1 $\triangle:: U R A 5 / T O R 1$ mutant strain was generated by PCR overlap using six primers as outlined (Fig. 1). The $5^{\prime}$ end of the TOR1 gene was amplified with primers JOHE4337 (no. 1) (CACTGAGTTGGAGTATTCTAC) and JOHE5850 (no. 3) (CATGGTCATAGCTGTTTCCTCGCCAAAGACGTAGAAGGC) and the $3^{\prime}$ end of TOR1 with primers JOHE5851 (no. 4) (ACTGGCCGTCGTTTTACGATAGACTCGAGGACGAGC) and JOHE4413 (no. 6) (GTCACCAAAGTAGTGCTTTG) and the URA5 selectable marker with primers JOHE5849 (no. 2) (GCCTTCTACGTCTTTGGCGAGGAAACAGCTATGACCATG) and JOHE5852 (no. 5) (GCTCGTCCTCGAGTCTATCGTAAAACGACGGCCAGT). The $U R A 5$ fragment in this disruption was amplified from plasmid pRCD69, which contains the C. neoformans serotype D URA5 sequence in the pCR2.1-TOPO vector, and the primers were designed to amplify from the vector sequence. The three amplified products were run on a $0.6 \%$ agarose gel and extracted together using the Qiaquik column method (Qiagen) and used as templates for the overlap reaction. Primers JOHE6209 (no. 1) and JOHE4413 (no. 6) were used to overlap the three first-round products to yield the tor $1 \Delta: U R A 5$ allele. The PCR product was gel-purified, extracted and transformed directly into the C. neoformans serotype $\mathrm{D}$ diploid strain RAS008 by biolistic transformation.

The mpk1 $1 \Delta: U R A 5$ mutant strain was generated by PCR overlap using six primers as outlined (Fig. 1). The $5^{\prime}$ end of the MPK1 gene was amplified with primers JOHE7288 (no. 1) (ACTAGGCGTGCCATTGTTTAC) and JOHE7418 (no. 3) (GGTCGAGCAACTTCGCTCAGGATTGCGTGCCGGACAGTG), the 3' of MPK1 with primers JOHE7419 (no. 4) (CCACCTCCTGGAGGCAAGCACGATGATTATTAGTCTTGC) and JOHE7289 (no. 6) (GCGGACTGGGCAGGAGAAGC), and the URA5 selectable marker with primers JOHE7417 (no. 2) (CACTGTCCGGCACGCAATCCTGAGCGAAGTTGCTCGACC) and JOHE7420 (no. 5) (GCAAGACTAATAATCATCGTGCTTGCCTCCAGGAGGTGG). The three amplified products were run on a $0.6 \%$ agarose gel and extracted together using the Qiaquik column method (Qiagen) and were used as templates for the overlap reaction. Primers JOHE7288 (no. 1) and JOHE7289 (no. 6) were used to overlap the three first-round products to yield the $m p k 1 \Delta:: U R A 5$ disruption allele. This amplified product was gel-purified, extracted and directly transformed into the serotype D ura5 strain JEC43 by biolistic transformation.

Overlap primer design. Overlap oligos were approximately $35-40$ bp in length. Primers were obtained from Integrated DNA Technology and were not PAGE-purified. In the STE11 $\alpha$ and MPK1 deletions, the portion of the oligo corresponding to the selectable marker flanked the C. neoformans URA5 gene. In the TOR 1 disruption, the portion of the oligo corresponding to the selectable marker was designed to amplify from a plasmid containing URA5. This was done so that any selectable marker cloned into the vector could be easily inserted into the disruption cassette.

The sequence of primer 3 was designed to be completely complementary to the sequence of primer 2 , and the sequence of primer 4 was complementary to the sequence of primer 5 as outlined in Fig. 1. This strategy generates fragments with approximately $40 \mathrm{bp}$ of overlap for the final PCR reaction. The 3 ' ends of primer 3 and primer 4 were designed so that the 


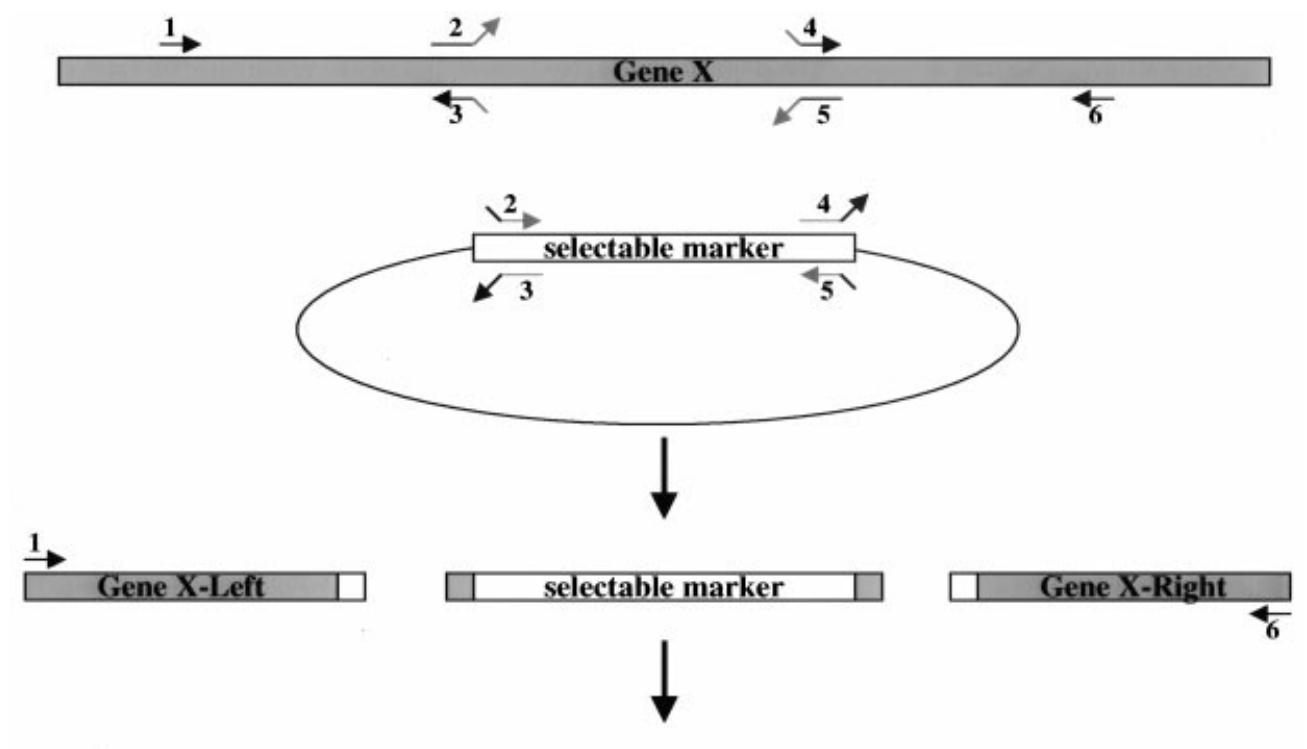

\begin{tabular}{l|l|l} 
Gene X-Left & selectable marker & Gene X-Right
\end{tabular}

Fig. 1. The PCR overlap procedure. A gene deletion allele is constructed by PCR as follows: the $5^{\prime}$ end of the gene to be deleted (Gene X) is amplified with primers 1 and 3 to generate fragment Gene X-Left, the $3^{\prime}$ end of Gene $X$ is amplified with primers 4 and 6 to generate fragment Gene X-Right, and the selectable marker is amplified with primers 2 and 5 . The amplified products are then used as templates for an overlap reaction using primers 1 and 6 . Primers 2 and 3 and primers 4 and 5, respectively, are complementary to one another and will therefore allow primers 1 and 6 to overlap the three first-round products into a linear PCR-amplified GeneX::selectable marker deletion allele.

melting temperature of the oligo fragment corresponding to the targeted gene was similar to the melting temperatures of primers 1 and 6, respectively.

Transformations. Biolistic transformations were performed by the method previously described by Toffaletti \& Perfect (1994) using a Bio-Rad model PDS-1000/He biolistic particle delivery system. Transformants were selected on defined medium (Sherman, 1991) lacking uracil and containing $1 \mathrm{M}$ sorbitol as an osmotic stabilizer.

PCR amplification. All PCR amplifications were performed using a Perkin-Elmer Applied Biosystems 9600 thermocycler with Extaq polymerase (Takara). The initial amplification reactions consisted of 35 cycles of $10-30 \mathrm{~s}$ at $95^{\circ} \mathrm{C}, 10-30 \mathrm{~s}$ at $55^{\circ} \mathrm{C}$ and $1 \mathrm{~min} \mathrm{~kb}^{-1}$ at $72{ }^{\circ} \mathrm{C}$ with an initial denaturation of $2 \mathrm{~min}$ at $95^{\circ} \mathrm{C}$ and a final extension of $5 \mathrm{~min}$ at $72^{\circ} \mathrm{C}$. Importantly, denaturation and annealing times were limited to $10-15 \mathrm{~s}$ during the final overlap amplification, which reproducibly increases the yield of specific overlap products.

The first-round PCR of the STE11 $\alpha$ disruption consisted of an initial denaturation of $2 \mathrm{~min}$ at $95^{\circ} \mathrm{C}$, followed by 35 cycles of $15 \mathrm{~s}$ at $95^{\circ} \mathrm{C}, 15 \mathrm{~s}$ at $53^{\circ} \mathrm{C}$ and $1.5 \mathrm{~min}$ at $72^{\circ} \mathrm{C}$, and was completed with a final extension of $5 \mathrm{~min}$ at $72{ }^{\circ} \mathrm{C}$. The final round of PCR for the STE11 $\alpha$ gene disruption consisted of an initial denaturation of 2 min at $95^{\circ} \mathrm{C}$, followed by 35 cycles of $15 \mathrm{~s}$ at $95^{\circ} \mathrm{C}, 15 \mathrm{~s}$ at $53^{\circ} \mathrm{C}$ and $4.5 \mathrm{~min}$ at $72^{\circ} \mathrm{C}$, and concluded with a final extension of $5 \mathrm{~min}$ at $72^{\circ} \mathrm{C}$.

In the final PCR, the three PCR products generated in the first PCR were added in roughly equimolar amounts. The quantity of these products yielding the most efficient overlap in the final PCR varied between constructs, so a gradient of first-round products was added to the reaction (generally $1 \mathrm{ng}, 10 \mathrm{ng}$ and $50 \mathrm{ng}$ total template) to obtain the most efficient overlap PCR.
The PCR samples were visualized using standard DNA electrophoretic techniques (Sambrook et al., 1989).

Southern blot analysis. Genomic DNA was isolated from JEC21 and JEC20 wild-type, ste $11 \alpha$ and mpk1 mutant strains by the method of Pitkin et al. (1996). Twenty micrograms genomic DNA was digested with the appropriate enzymes and electrophoresed on a $0.8 \%$ TBE agarose gel. Transfer, hybridization and autoradiography were performed as described by Sambrook et al. (1989). Fragments of the STE11 $\alpha$ and MPK1 genomic ORFs were used as probes for Southern blot hybridization, using $\left[\alpha^{32} \mathrm{P}\right] \mathrm{dCTP}$ (Amersham) and the Prime-It II random primed labelling kit (Stratagene).

\section{RESULTS}

\section{Targeted disruption of the STE11 $\alpha$ gene}

The $S T E 11 \alpha$ gene encodes a mating type-specific MEK kinase homologue that is related to the Ste11 kinase of $S$. cerevisiae, a component of the pheromone-sensing mitogen-acitvated protein (MAP) kinase cascade. The C. neoformans STE11 $\alpha$ gene was identified by its location in the $M A T \alpha$ locus adjacent to the first pheromone gene discovered in C. neoformans by Moore \& Edman (Clarke et al., 2001; Moore \& Edman, 1993). To perform epistasis analysis with other members of this MAP kinase cascade, we made a ste $11 \alpha$ mutant strain by homologous integration of a disruption allele. We used PCR to generate a ste $11 \alpha:$ :URA5 disruption allele. Six oligonucleotide primers (numbered 1-6) were directed against the STE11 $\alpha$ ORF and the URA5 selectable marker (strategy outlined in Fig. 1). Separate PCR 

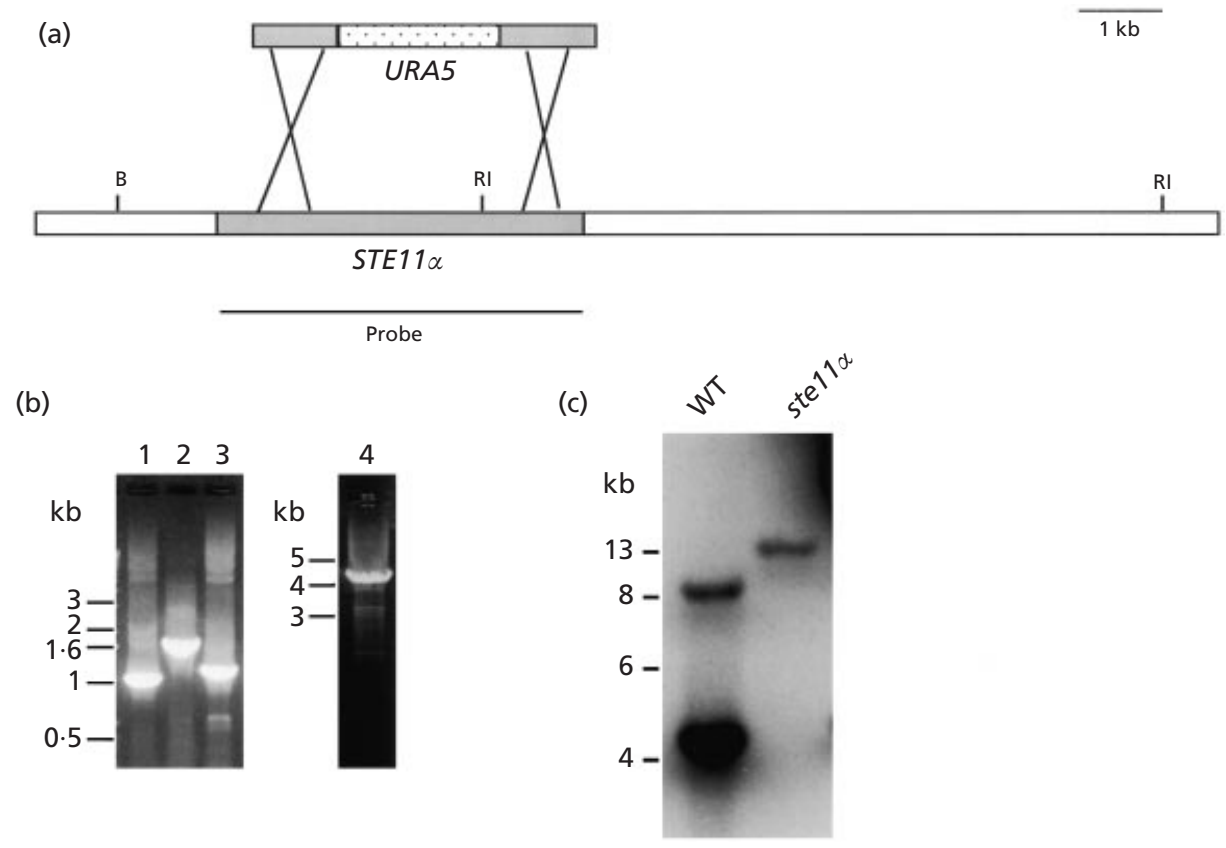

Fig. 2. Deletion of the STE11 $\alpha$ gene. (a) The STE11 $\alpha$ gene was deleted by replacing a portion of the STE $11 \alpha$ ORF with the URA5 selectable marker by PCR overlap deleting $2 \cdot 2 \mathrm{~kb}$ of STE11 $\alpha$. (b) The 5 ' region of STE11 $\alpha$ (lane 1), the URA5 selectable marker (lane 2), and the $3^{\prime}$ region of STE11 $\alpha$ (lane 3) were amplified as described (Methods). The three products were used as templates to amplify the ste11 $\alpha:$ URA5 deletion allele (lane 4). (c) Southern analysis was performed to confirm the gene deletion. Genomic DNA from wild-type MAT $\alpha$ (WT) and the ste11 $\alpha$ mutant strain was digested with $B a m \mathrm{HI}(\mathrm{B})$ and EcoRI (RI), subjected to Southern blot analysis, and probed with a $4 \mathrm{~kb}$ PCR fragment of the STE11 $\alpha$ gene. Positions of DNA size markers are shown on the left.

amplification with these primers yielded three overlapping products: the $5^{\prime}$ end of the STE11 $\alpha$ gene, the URA5 selectable marker, and the $3^{\prime}$ end of the STE11 $\alpha$ gene (Fig. 2a, b, lanes 1-3). In a second round of amplification, the three products were used as templates to generate a single linear disruption allele that contained the URA5 gene flanked by portions of the STE11 $\alpha$ gene (Fig. 2b, lane 4). This linear PCR product was directly transformed into a serotype D MAT $\alpha$ ura5 strain of C. neoformans (JEC43) by biolistic transformation, and $\mathrm{Ura}^{+}$colonies were selected. Screening by PCR amplification revealed three mutant strains out of 48 independent $\mathrm{Ura}^{+}$colonies $(6.3 \%)$ (Table 1), which was confirmed by Southern blot analysis (Fig. 2c). Each of the ste $11 \alpha$ mutant strains exhibited the same level of sterility in a cross with a wild-type MATa strain, and this was consistent with results obtained with mutants lacking other members of the MAP kinase cascade (R. C. Davidson \& J. Héitman, unpublished).

\section{Targeted disruption of the TOR1 gene}

The TOR1 gene encodes a homologue of the Tor kinases of $S$. cerevisiae. To obtain functional information about this gene, we took a gene-deletion approach. The gene was originally identified in serotype A (Cruz et al., 1999), and the serotype D sequence was then partially assembled from the Stanford and TIGR databases by comparison of serotype A and serotype D sequences. Six primers (numbered 1-6) were constructed to amplify the $5^{\prime}$ and $3^{\prime}$ portions of the TOR 1 gene and the selectable marker, URA5 (Fig. 3a, b, lanes 1-3). These three products and primers 1 and 6 were used to amplify a single linear disruption allele that consisted of URA5 flanked by $\sim 1 \mathrm{~kb}$ of TOR 1 gene sequence on each side (Fig. 3b, lane 4). The linear overlap PCR product was gel-purified and used directly for transformation into a haploid recipient strain of C. neoformans. No homologous recombination events were obtained out of approximately $200 \mathrm{Ura}^{+}$isolates screened, indicating that TOR1 might be an essential gene in C. neoformans.

To establish whether TOR1 is essential or not, we sought to isolate tor $1 /$ TOR1 heterozygous mutants using the recently discovered stable, congenic diploid strains of C. neoformans (Sia et al., 2000). A ura5/ura5 diploid strain of C. neoformans (RAS008) was transformed with the linear tor1 $1 \Delta:$ URA5 PCR-generated disruption allele, and $\mathrm{Ura}^{+}$transformants were selected. Using PCR amplification and digestion with the SalI restriction enzyme, which specifically cleaves the mutant allele but not the wild-type at a pair of recognition sites present in the URA5 gene (Fig. 3c), one tor $1:: U R A 5 /$ TOR 1 strain was identified out of $56 \mathrm{Ura}^{+}$transformants screened $(1 \cdot 8 \%)$ (Table 1$)$. These results indicate that PCR-generated disruption constructs can be used to target genes in the laboratory-constructed congenic 
Table 1. Genes disrupted using PCR-generated targeting alleles

\begin{tabular}{|c|c|c|c|}
\hline Gene & Strain (genotype) & Frequency* $(\%)$ & Homology $†$ \\
\hline ste $11 \alpha \Delta:: U R A 5$ & JEC43 (Sero D MAT $\alpha$ ura5) & 2 & $1040 \ldots 1180$ \\
\hline tor $1 \Delta:: U R A 5$ & RAS008 (Sero D MATa/a ura5/ura5) & 2 & $1222 \ldots 1030$ \\
\hline$m p k 1 \Delta:: U R A 5$ & JEC43 (Sero D MAT $\alpha$ ura5) & 10 & $628 \ldots 835$ \\
\hline ste $12 \alpha \Delta:: U R A 5$ & JEC155 (Sero D ade2-27 ura5) & 10 & $1476 \ldots 1661$ \\
\hline tlk1 $1 \Delta:$ URA5 & JEC34 (Sero D MATa ura5) & 4 & $789 \ldots 956$ \\
\hline$f h b 1 \Delta:: U R A 5$ & JEC43 (Sero D MAT $\alpha$ ura5) & 33 & $1044 \ldots 1296$ \\
\hline$f h b 1 \Delta:: U R A 5$ & H99 5-FOA ${ }^{\mathrm{r}}$ (Sero A MAT $\alpha$ ura5) & 22 & $682 \ldots 1113$ \\
\hline gno1A::NAT1 & JEC34 (Sero D MATa ura5) & $23 \neq$ & $792 \ldots 656$ \\
\hline gno1 $1:$ :NAT1 & H99 5-FOA ${ }^{\mathrm{r}}$ (Sero A MAT $\alpha$ ura5) & $77 \neq$ & $549 \ldots 656$ \\
\hline $\operatorname{prf1\Delta ::URA5}$ & JEC43 (Sero D MAT $\alpha$ ura5) & 87 & $800 \ldots 361$ \\
\hline pka2A::URA5 & JEC43 (Sero D MAT $\alpha$ ura5) & 3 & $530 \ldots 860$ \\
\hline $\operatorname{sxi1} \alpha \Delta:: U R A 5$ & JEC43 (Sero D MAT $\alpha$ ura5) & 10 & $1102 \ldots 1007$ \\
\hline sxi1 $\alpha \Delta:: U R A 5$ & RAS009 (Sero D MATa/ ura5/ura5) & 4 & $1102 \ldots 1007$ \\
\hline $\operatorname{sxi1} \alpha \Delta:$ :NAT1 & JEC43 (Sero D MAT $\alpha$ ura5) & 10 & $1103 \ldots 1014$ \\
\hline $\operatorname{sxi1} \alpha \Delta:$ :NAT1 & RAS009 (Sero D MATa/ $\alpha$ ura $5 /$ ura5) & ND & $1103 \ldots 1014$ \\
\hline gpa1s::URA5 & H99 5-FOA ${ }^{\mathrm{r}}($ Sero A MAT $\alpha$ ura5) & 12 & $650 \ldots 540$ \\
\hline $\operatorname{crg} 1 \Delta:: U R A 5$ & PPW28 (Sero A MAT $\alpha$ ade2 sch9D::ADE2 ura5) & 10 & $590 \ldots 605$ \\
\hline
\end{tabular}

*Frequency refers to the homologous recombination frequency obtained. ND, Not determined.

† Homology refers to the amount of the $5^{\prime}$ (left) and 3' (right) regions of each gene incorporated into the disruption alleles in base pairs. $\ddagger$ In these cases, this is the frequency of homologous recombination in isolated stable transformants.

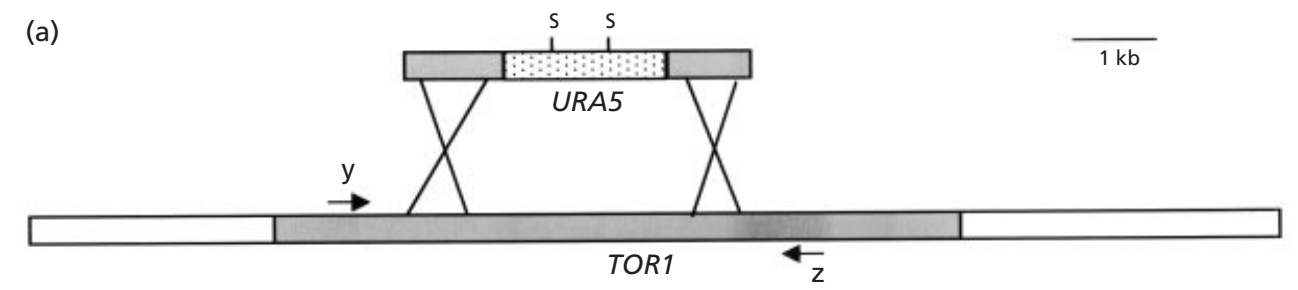

(b)

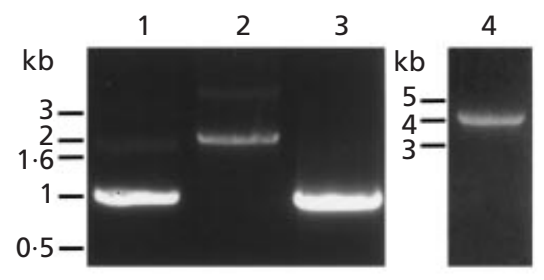

(c)

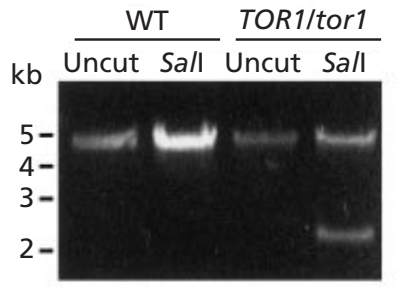

Fig. 3. Deletion of the TOR1 gene. (a) The TOR1 gene was deleted by replacing a portion of the TOR1 ORF with the URA5 selectable marker by PCR overlap. (b) The $5^{\prime}$ region of TOR1 (lane 1), the URA5 selectable marker (lane 2), and the $3^{\prime}$ region of TOR1 (lane 3) were amplified as described (Methods). The three products were used as templates to amplify the tor1::URA5 deletion allele (lane 4). (c) PCR analysis was performed to confirm the heterozygous gene deletion. Genomic DNA from wild-type MAT $\alpha$ (WT) and the tor1/TOR1 mutant strain was PCR-amplified with primers $y$ and $z$ and digested with Sall (S), which specifically digests the mutant allele but not the wild-type allele. Two of the fragments generated by the digestion of the mutant disruption allele were approximately the same size and thus appear as a single $2 \cdot 1 \mathrm{~kb}$ band. The smaller Sall-Sall fragment inside the URA5 sequence of approximately $650 \mathrm{bp}$ is not shown in this figure.

diploid strains, and provide evidence that TOR1 is an essential gene, since it could be disrupted in a diploid, but not a haploid, strain of C. neoformans. We do note that gene disruption in the congenic serotype D dip- loid strains typically results in a lower frequency of homologous recombination $(1-5 \%)$ than that commonly observed in haploid recipient strains $(2-25 \%$ on average). 


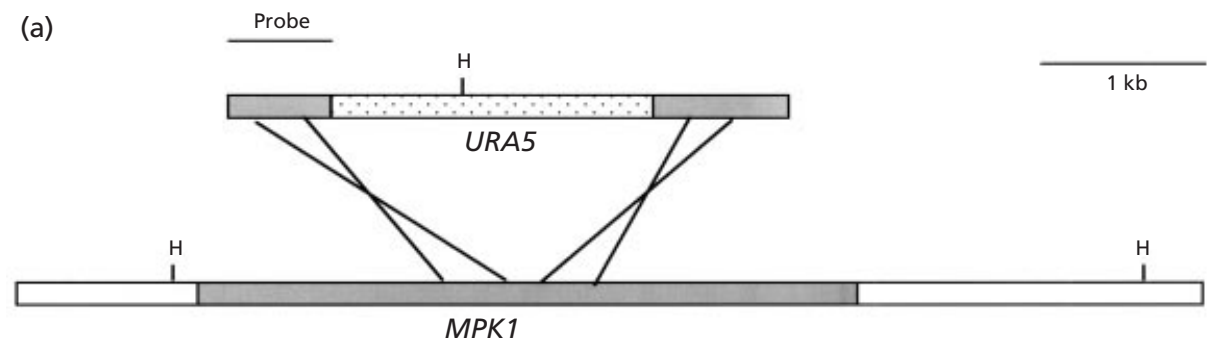

(b)

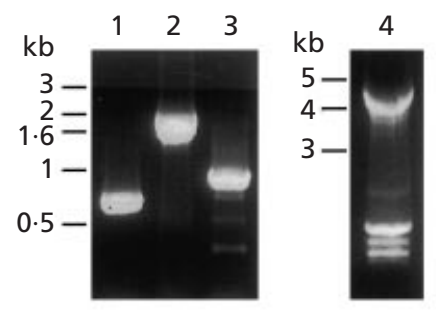

(c)

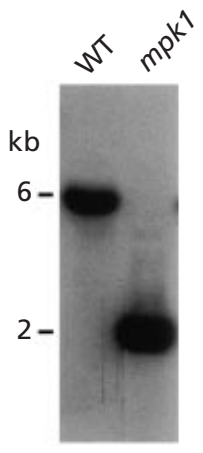

Fig. 4. Disruption of the MPK1 gene. (a) The MPK1 gene was disrupted by introducing the URA5 selectable marker into the MPK1 ORF by PCR overlap. (b) The 5 ' region of MPK1 (lane 1), the URA5 selectable marker (lane 2), and the 3' region of MPK1 (lane 3) were amplified as described (Methods). The three products were used as templates to amplify the mpk1::URA5 deletion allele (lane 4). (c) Southern analysis was performed to confirm the gene deletion. Genomic DNA

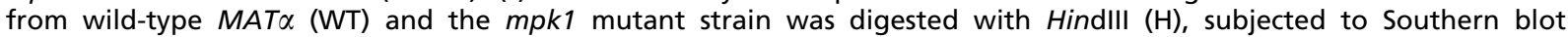
analysis, and probed with a $1 \mathrm{~kb}$ PCR fragment of the $5^{\prime}$ end of the MPK1 gene. Positions of DNA size markers are shown on the left.

\section{Targeted disruption of the MPK1 gene}

The gene encoding the MAP kinase homologue Mpk1 was identified by comparative genomics using the Stanford C. neoformans genome sequence database (P. R. Kraus and others, unpublished). A complete sequence of the putative gene was assembled using the Stanford sequence as well as that from the TIGR C. neoformans sequencing project. To assess the function of this MAP kinase homologue, we generated an allele for gene disruption using PCR overlap. As described above, primers were directed against the $5^{\prime}$ and $3^{\prime}$ flanking regions of the MPK1 gene and the URA5 selectable marker, and three products were amplified (Fig. 4a, b, lanes 1-3). These products were overlapped into a single product with the portions of the MPK1 gene

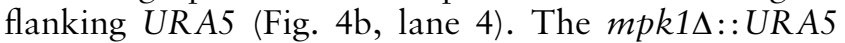
PCR-generated disruption allele was transformed into a serotype D ura5 strain of C. neoformans (JEC43), and

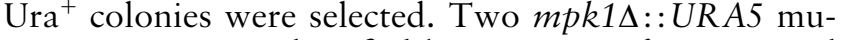
tant strains were identified by PCR out of 20 screened $(10 \%)$ (Table 1), which was confirmed by a Southern blot (Fig. 4c).

\section{Summary of results}

Together, these results indicate that PCR overlap can be used to generate targeting alleles for gene disruption in C. neoformans. Successful PCR overlap amplification products have been obtained in each case for which it has been attempted (Table 1). However, in some cases, obtaining sufficient product for transformation has required manipulation of PCR parameters, particularly when transforming diploid strains, which typically require more transforming DNA $(3-5 \mu \mathrm{g})$ to obtain transformants. For example, reducing the denaturation and annealing times to $15 \mathrm{~s}$ or less has increased product yield dramatically in several cases, particularly when using the dominant selectable marker NAT1. Additionally, to maximize product, it is important to maintain relatively equimolar amounts of the templates in the overlap reaction. In all, we have obtained 17 gene disruptions using this PCR overlap technique to generate targeting alleles. Thus, we show that the PCR overlap technique is generally applicable and can be used with a variety of recipient strains as well as with both auxotrophic and dominant selectable markers (Table 1).

\section{DISCUSSION}

C. neoformans is an important human pathogenic fungus. As a genetically tractable organism with welldeveloped molecular biology and animal models, and a nearly completed genomic sequence that is publicly available, C. neoformans is an excellent model for the study of fungal pathogenesis (Casadevall \& Perfect, 1998). However, efficient use of the sequence data 
requires implementation of improved molecular techniques such as gene disruption. In many model systems, PCR has replaced cloning as a faster, more efficient method of generating disruption alleles. Generation of disruption constructs in S. cerevisiae is performed almost exclusively by the technique involving large synthetic oligonucleotides that contain $40 \mathrm{bp}$ of homology to the gene of interest followed by $20 \mathrm{bp}$ of homology to the selectable marker (Eberhardt \& Hohmann, 1995; Lorenz et al., 1995; Wach, 1996; Wach et al., 1994). The selectable marker is then amplified and the PCR product used to directly transform and generate the mutant strain (Tong et al., 2001). This technique was also recently implemented for producing mutant strains in the human pathogen Candida albicans (Wilson et al., 1999). However, in Cryptococcus neoformans, a larger region of homology is required for integration and thus cannot be incorporated into synthetic oligonucleotides.

We describe a modified use of the PCR overlap technique originally described by Ho et al. (1989) and Horton et al. (1989) to generate disruption constructs without the need for cloning. This method can be used to generate both partial and complete deletions of the targeted gene's ORF. Using this method, we have disrupted three genes, STE11 $\alpha$ (encoding a MAP kinase kinase kinase homologue), TOR1 (encoding a Tor kinase homologue) and MPK1 (encoding a MAP kinase homologue), at efficiencies consistent with previous studies (Davidson et al., 2000). We also report the disruption of 11 other genes using the same method and a variety of recipient strains, including the recently described serotype D diploids (Sia et al., 2000). Moreover, both the auxotrophic URA5 and dominant NAT1 selectable markers were used, which allows generation of double mutant strains or the use of prototrophic strains. In all cases, the efficiency of targeted integration was in the range 2-10\% or higher, consistent with previous studies using biolistic transformation in C. neoformans (Alspaugh et al., 1997; Davidson et al., 2000; Toffaletti et al., 1993; Wang et al., 2000). These findings indicate that generation of constructs by two rounds of PCR does not seem to inhibit homologous recombination significantly.

Additional screening tests can make the PCR-disruption method even more efficient. Jennifer Lodge and coworkers recently found that a significant proportion of initial transformants are unstable, in accord with earlier results (Edman \& Kwon-Chung, 1990), and by implementing an initial screening step for stable transformants they found that the efficiency of homologous targeting can be increased (Nelson et al., 2002). We have confirmed these observations in the background of our PCR-based approach to gene disruption and found that approximately $33 \%$ of transformants are stable. In the case of the gno1::NAT1 disruption in strain JEC34, the frequency of homologous integration was increased from 10 to $23 \%$, and, in the case of the gno1::NAT1 disruption in strain H99, from 28 to $77 \%$, by first screening for stable transformants. Thus, implementing this important advance of Lodge and coworkers, together with the PCR overlap approaches described here, should result in even more efficacious gene-disruption frequencies.

Implementation of this PCR-based gene-disruption technique will preclude the generation of mutant strains from being the rate-limiting step in performing genetic analyses. The recently reported RNAi and antisense methods for disrupting gene function and the ability to generate panels of strains containing randomly inserted signature tags should also aid in accelerating the analysis of gene function (Gorlach et al., 2002; Liu et al., 2002; Nelson et al., 2001). However, the PCR-based method proposed here has the potential to be applied to situations in which the ability to alter targeted genomic sequence is necessary. For instance, larger deletions that span multiple genes can be performed using this method. In addition to disruption mutations, this technique can also be applied to the efficient generation of other targeted insertions, including site-directed mutations and the insertion of regulatable promoters or epitope tags.

In conclusion, we show that this PCR-based genedisruption approach is generally applicable for different genes using a variety of strains and genetic markers, and this should allow more efficient analysis of gene function in cases where the complete sequence is available.

\section{ACKNOWLEDGEMENTS}

We thank John Perfect, Jenifer Görlach, Andy Alspaugh, Cristina Cruz, Connie Nichols and Klaus Lengeler for valuable technical advice and scientific discussions, Debbie Fox for reviewing the manuscript, and Jennifer Lodge for sharing data with us prior to publication. These studies were supported, in part, by P01 grant AI44975 to the Duke University Mycology Research Unit, R01 grants AI39115, AI50438, AI50115 (J.H.) and a minority supplement, AI39115S1 (M. J.B.), all from the NIAID. C. M.H. is supported by a Damon Runyon Cancer Research Fund Fellowship (DRG-1694). J.H. is an associate investigator of the Howard Hughes Medical Institute and a Burroughs Wellcome Scholar in molecular pathogenic mycology. Some of the genomic sequences used in this paper were obtained from the C. neoformans Genome Project, Stanford Genome Technology Center, funded by the NIAID/NIH under cooperative agreement U01 AI47087, and The Institute for Genomic Research, funded by the NIAID/NIG under cooperative agreement U01 AI48594.

\section{REFERENCES}

Alspaugh, J. A., Perfect, J. R. \& Heitman, J. (1997). Cryptococcus neoformans mating and virulence are regulated by the G-protein $\alpha$ subunit GPA1 and cAMP. Genes Dev 11, 3206-3217.

Alspaugh, J. A., Cavallo, L. M., Perfect, J. R. \& Heitman, J. (2000a). RAS1 regulates filamentation, mating and growth at high temperature of Cryptococcus neoformans. Mol Microbiol 36, 352-365.

Alspaugh, J. A., Davidson, R. C. \& Heitman, J. (2000b). Morphogenesis of Cryptococcus neoformans. In Dimorphism in Human Pathogenic and Apathogenic Yeasts, pp. 217-238. Edited by J. F. Ernst \& A. Schmidt. Basel: Karger.

Baudin, A., Ozier-Kalogeropoulos, O., Denouel, A., Lacroute, F. \& 
Cullin, C. (1993). A simple and efficient method for direct gene deletion in Saccharomyces cerevisiae. Nucleic Acids Res 21, 3329-3330.

Casadevall, A. \& Perfect, J. R. (1998). Cryptococcus neoformans. Washington: ASM Press.

Chen, S. C., Wright, L. C., Golding, J. C. \& Sorrell, T. C. (2000). Purification and characterization of secretory phospholipase B, lysophospholipase and lysophospholipase/transacylase from a virulent strain of the pathogenic fungus Cryptococcus neoformans. Biochem J 347, 431-439.

Clarke, D. L., Woodlee, G. L., McClelland, C. M., Seymour, T. S. \& Wickes, B. L. (2001). The Cryptococcus neoformans STE11 $\alpha$ gene is similar to other fungal mitogen-activated protein kinase kinase kinase (MAPKKK) genes but is mating type specific. Mol Microbiol 40, 200-213.

Cox, G. M., Mukherjee, J., Cole, G. T., Casadevall, A. \& Perfect, J. R. (2000). Urease as a virulence factor in experimental cryptococcosis. Infect Immun 68, 443-448.

Cox, G. M., McDade, H. C., Chen, S. C. A. \& 8 other authors (2001). Extracellular phospholipase activity is a virulence factor for Cryptococcus neoformans. Mol Microbiol 39, 166-175.

Cruz, M. C., Cavallo, L. M., Görlach, J. M., Cox, G., Perfect, J. R., Cardenas, M. E. \& Heitman, J. (1999). Rapamycin antifungal action is mediated via conserved complexes with FKBP12 and TOR kinase homologs in Cryptococcus neoformans. Mol Cell Biol 19, 4101-4112.

Cruz, M. C., Sia, R. A. L., Olson, M., Cox, G. M. \& Heitman, J. (2000). Comparison of the roles of calcineurin in physiology and virulence in serotype D and serotype A strains of Cryptococcus neoformans. Infect Immun 68, 982-985.

Davidson, R. C., Cruz, M. C., Sia, R. A. L., Allen, B. M., Alspaugh, J. A. \& Heitman, J. (2000). Gene disruption by biolistic transformation in serotype D strains of Cryptococcus neoformans. Fungal Genet Biol 29, 38-48.

Eberhardt, I. \& Hohmann, S. (1995). Strategy for deletion of complete open reading frames in Saccharomyces cerevisiae. Curr Genet 27, 306-308.

Edman, J. C. \& Kwon-Chung, K. J. (1990). Isolation of the URA5 gene from Cryptococcus neoformans var. neoformans and its use as a selective marker for transformation. Mol Cell Biol 10, $4538-4544$.

Fox, D. S., Cruz, M. C., Sia, R. A. L., Ke, H., Cox, G. M., Cardenas, M. E. \& Heitman, J. (2001). Calcineurin regulatory subunit is essential for virulence and mediates interactions with FKBP12FK506 in Cryptococcus neoformans. Mol Microbiol 39, 835-849.

Goldman, D., Lee, S. C. \& Casadevall, A. (1994). Pathogenesis of pulmonary Cryptococcus neoformans infection in the rat. Infect Immun 62, 4755-4761.

Gorlach, J. M., McDade, H. C., Perfect, J. R. \& Cox, G. M. (2002). Antisense repression in Cryptococcus neoformans as a laboratory tool and potential antifungal strategy. Microbiology 148, 213219

Heitman, J., Casadevall, A., Lodge, J. K. \& Perfect, J. R. (1999). The Cryptococcus neoformans genome sequencing project. Mycopathologia 148, 1-7.

Ho, S. N., Hunt, H. D., Horton, R. M., Pullen, J. K. \& Pease, L. R. (1989). Site-directed mutagenesis by overlap extension using the polymerase chain reaction. Gene 77, 51-59.

Horton, R. M., Hunt, H. D., Ho, S. N., Pullen, J. K. \& Pease, L. R. (1989). Engineering hybrid genes without the use of restriction enzymes: gene splicing by overlap extension. Gene 77, 61-68.

Hua, J., Meyer, J. D. \& Lodge, J. K. (2000). Development of positive selectable markers for the fungal pathogen Cryptococcus neoformans. Clin Diagn Lab Immunol 7, 125-128.

Kwon-Chung, K. J. (1975). A new genus, Filobasidiella, the perfect state of Cryptococcus neoformans. Mycologia 67, 1197-1200.

Kwon-Chung, K. J. (1976). Morphogenesis of Filobasidiella neoformans, the sexual state of Cryptococcus neoformans. Mycologia 68, 821-833.

Kwon-Chung, K. J. \& Bennett, J. E. (1992). Cryptococcosis. In Medical Mycology, pp. 397-446. Malvern, PA: Lea \& Febiger.

Kwon-Chung, K. J., Polacheck, I. \& Popkin, T. J. (1982). Melaninlacking mutants of Cryptococcus neoformans and their virulence for mice. J Bacteriol 150, 1414-1421.

Liu, H., Cottrell, T. R., Pierini, L. M., Goldman, W. E. \& Doering, T. L. (2002). RNA interference in the pathogenic fungus Cryptococcus neoformans. Genetics 160, 463-470.

Lorenz, M. C., Muir, R. S., Lim, E., McElver, J., Weber, S. C. \& Heitman, J. (1995). Gene disruption with PCR products in Saccharomyces cerevisiae. Gene 158, 113-117.

McDade, H. C. \& Cox, G. M. (2001). A new dominant selectable marker for use in Cryptococcus neoformans. Med Mycol 39, 151-154.

Mitchell, T. G. \& Perfect, J. R. (1995). Cryptococcosis in the era of AIDS - 100 years after the discovery of Cryptococcus neoformans. Clin Microbiol Rev 8, 515-548.

Moore, T. D. E. \& Edman, J. C. (1993). The $\alpha$-mating type locus of Cryptococcus neoformans contains a peptide pheromone gene. Mol Cell Biol 13, 1962-1970.

Nelson, R. T., Hua, J., Pryor, B. \& Lodge, J. K. (2001). Identification of virulence mutants of the fungal pathogen Cryptococcus neoformans using signature-tagged mutagenesis. Genetics 157, 935-947.

Nelson, R. T., Pryor, B. A. \& Lodge, J. K. (2002). Sequence length required for homologous recombination in Cryptococcus neoformans. Fungal Genet Biol (in press).

Odom, A., Muir, S., Lim, E., Toffaletti, D. L., Perfect, J. \& Heitman, J. (1997). Calcineurin is required for virulence of Cryptococcus neoformans. EMBO J 16, 2576-2589.

Perfect, J. R., Lang, S. D. R. \& Durack, D. T. (1980). Chronic cryptococcal meningitis: a new experimental model in rabbits. Am J Pathol 101, 177-194.

Pitkin, J. W., Panaccione, D. G. \& Walton, J. D. (1996). A putative cyclic peptide efflux pump encoded by the TOXA gene of the plant-pathogenic fungus Cochliobolus carbonum. Microbiology 142, 1557-1565.

Sambrook, J., Fritsch, E. F. \& Maniatis, T. (1989). Molecular Cloning: a Laboratory Manual. Cold Spring Harbor, NY: Cold Spring Harbor Laboratory.

Sanfelice, F. (1894). Contributo alla morfologia e biologia dei blastomiceti che si sviluppano nei succhi di alcuni frutti. Ann Igien 4, 463-495.

Sherman, F. (1991). Getting started with yeast. Methods Enzymol 194, 3-21.

Sia, R. A., Lengeler, K. B. \& Heitman, J. (2000). Diploid strains of the pathogenic basidiomycete Cryptococcus neoformans are thermally dimorphic. Fungal Genet Biol 29, 153-163.

Sudarshan, S., Davidson, R. C., Heitman, J. \& Alspaugh, J. A. (1999). Molecular analysis of the Cryptococcus neoformans ADE2 gene, a selectable marker for transformation and gene disruption. Fungal Genet Biol 27, 36-48.

Toffaletti, D. L. \& Perfect, J. R. (1994). Biolistic DNA delivery for Cryptococcus neoformans transformation. In Molecular Biology 
of Pathogenic Fungi: a Laboratory Manual, pp. 303-308. Edited by B. Maresca \& G. S. Kobayashi. New York: Telos Press.

Toffaletti, D. L., Rude, T. H., Johnston, S. A., Durack, D. T. \& Perfect, J. R. (1993). Gene transfer in Cryptococcus neoformans by use of biolistic delivery of DNA. J Bacteriol 175, 1405-1411.

Tong, A. H. Y., Evangelista, M., Parsons, A. B. \& 10 other authors (2001). Systematic genetic analysis with ordered arrays of yeast deletion mutants. Science 294, 2364-2368.

Wach, A. (1996). PCR-synthesis of marker cassettes with long flanking homology regions for gene disruptions in S. cerevisiae. Yeast 12, 259-265.

Wach, A., Brachat, A., Pohlmann, R. \& Philippsen, P. (1994). New heterologous modules for classical or PCR-based gene disruptions in Saccharomyces cerevisiae. Yeast 10, 1793-1808.

Wang, P., Perfect, J. R. \& Heitman, J. (2000). The G-protein $\beta$ subunit GPB1 is required for mating and haploid fruiting in Cryptococcus neoformans. Mol Cell Biol 20, 352-362.

Wilson, R. B., Davis, D. \& Mitchell, A. P. (1999). Rapid hypothesis testing with Candida albicans through gene disruption with short homology regions. J Bacteriol 181, 1868-1874.

Received 27 February 2002; revised 27 March 2002; accepted 18 April 2002. 